\title{
¿CUÁl ES LA NATURALEZA DEL ÓRGANO QUE GENERA PLEITO PENDIENTE INTERNACIONAL ANTE LA COMISIÓN INTERAMERICANA DE DERECHOS HUMANOS?*
}

\author{
Andrés González Serrano**
}

Fecha de recibido: 16 de octubre de 2014

Fecha de aprobado: 10 de diciembre de 2014

Artículo de reflexión

Forma de citación: González A. (2015). ¿Cuál es la naturaleza del órgano que genera pleito pendiente internacional ante la Comisión Interamericana de Derechos Humanos? Revista Prolegómenos. Derechos y Valores, 18, 35, 189-202.

\section{Resumen}

El artículo describe lo que la Comisión Interamericana de Derechos Humanos entiende por la expresión "otro organismo internacional gubernamental", al analizar la admisibilidad de una petición individual en relación con la existencia o no de pleito pendiente internacional. A partir de una investigación básica, descriptiva y deductiva se aborda la pregunta ¿̇cuál es la calidad del órgano que genera pleito pendiente internacional ante la Comisión Interamericana de Derechos Humanos? El objetivo general es identificar las líneas de argumentación de la Comisión cuando un Estado propone la inadmisibilidad de una petición por la existencia de pleito pendiente internacional. Se concluye que se está en presencia de pleito pendiente internacional cuando se tramita de forma conjunta una petición ante otro órgano de protección de derechos humanos al cual el Estado haya concedido competencia, considerando además que el mandato de dicho órgano debe ser convencional y el procedimiento seguido por él adjudicativo, contencioso y contradictorio.

\section{Palabras claves:}

Comisión Interamericana de Derechos Humanos, pleito pendiente internacional, Organismo Internacional Gubernamental, mandato, procedimiento, inadmisibilidad.

\section{¿WHICH IS THE NATURE OF THE ORGAN THAT GENERATES INTERNATIONAL PENDING PROCEEDING BEFORE THE INTER-AMERICAN COMMISSION ON HUMAN RIGHTS?}

\begin{abstract}
The article describes what the Inter-American Commission on Human Rights meant by the term "another international governmental organization" when analyzing the admissibility of an indivi-
\end{abstract}

\footnotetext{
Artículo derivado del proyecto DER 1490 "Duplicidad de procedimientos internacionales. Una mirada desde el Sistema Interamericano", financiado por la Vicerrectoría de Investigaciones de la Universidad Militar Nueva Granada, UMNG (Vigencia 2014). Dicha investigación pertenece a la línea de investigación sobre "Derechos Humanos y Derecho Internacional Humanitario" del Grupo de Derecho Público, adscrito al Centro de Investigaciones Jurídicas Políticas y Sociales de la Facultad de Derecho de la UMNG.

** Docente de la Universidad Militar Nueva Granada. Investigador del Grupo de Derecho Público y de la línea de investigación de "Derechos Humanos y Derecho Internacional Humanitario" del Centro de Investigaciones Jurídicas, Políticas y Sociales de la Facultad de Derecho de la Universidad Militar Nueva Granada. Abogado Magna Cum Laude de la Universidad Militar Nueva Granada (Colombia). Especialista en Docencia Universitaria y Magíster en Derecho Administrativo de la Universidad Militar Nueva Granada (Colombia). Magíster en Protección Internacional de los Derechos Humanos de la Universidad de Alcalá (España). Cursando Doctorado en la Universidad de Alcalá (España). Correo electrónico: andres. gonzalez@unimilitar.edu.co.
} 
dual petition regarding the existence or not of an international pending case. Through a basic, descriptive and inferential research the article addresses the question cwhich is the nature of the organ that generates international pending proceeding before the Inter-American Commission on Human Rights? The overall objective is to identify the lines of argumentation of the Commission when a State proposes the inadmissibility of a request by the existence of international pending proceeding. As a result it is concluded that there is an international pending litigation when jointly filed a petition with other organ of human rights protection, which the State has granted competence and mandate is conventional and the proceedings before it is adjudicative, contentious and contradictory.

\title{
Key words:
}

Inter-American Commission on Human Rights, international pending proceeding, International Governmental Organization ,mandate, procedure, inadmissibility.

\section{O QUE É A QUALIDADE DO CORPO QUE GERA LITÍGIO PENDENTE INTERNACIONAL PERANTE A COMISSÃO DE DIREITOS HUMANOS?}

\begin{abstract}
Resumo
O artigo descreve o que a Comissão Inter-Americana de Direitos Humanos entende pelo termo "outra organismo internacional governamental", analisando a admissibilidade de uma petição individual sobre a existência ou não de Litígio Pendente Internacional. A partir de uma questão básica, descritiva e inferencial aborda-se pesquisa do que é a qualidade do órgão que gera Litígio Pendente Internacional perante a Comissão Interamericana de Direitos Humanos? $\mathrm{O}$ objetivo geral é identificar a linha de argumentação da Comissão quando um Estado propõe a inadmissibilidade de um pedido da existência de Litígio Pendente Internacional.

Como resultado, conclui-se que, na presença de um litígio pendente internacional quando apresentado conjuntamente uma petição pendente um órgão de proteção dos direitos humanos dos quais o Estado tenha concedido acompetência, considerando que o mandato deste órgão deve ser convencional e que, além disso, o processo judicante seguido para ele deve ser contencioso e contraditório.
\end{abstract}

\section{Palavras chave:}

Comissão Interamericana de Direitos Humanos, litigio pendente internacional,Organismo Internacional Governamental, mandato, procedimento, inadmissibilidade.

\section{INTRODUCCIÓN}

El presente artículo hace parte del Proyecto "La duplicidad de procedimientos internacionales. Una mirada desde el Sistema Interamericano de Protección de Derechos Humanos", investigación que pretende abordar y dar solución a la pregunta sobre ¿́cuáles son los requisitos de estimación de la excepción preliminar relativa a la duplicidad de procedimientos internacionales? El proyecto de investigación se construye sobre la necesidad de continuar con la tarea académica de determinar y establecer los diferentes espacios convencionales procedimentales; identificar y construir nichos citacionales; y crear líneas jurisprudenciales que permitan acercar a los defensores de derechos humanos a la forma cómo la Comisión Interamericana de Derechos 
Humanos (CIDH) aplica e interpreta los requisitos de procedibilidad establecidos en la Convención Americana sobre Derechos Humanos (CADH) en el sistema de peticiones individuales e interestatales.

A la fecha no se conoce iniciativa que pretenda abordar el tema objeto de estudio de la manera aquí propuesta, motivo por el cual el proyecto constituye un aporte al fortalecimiento y legitimidad del Sistema Interamericano. Sin embargo, es importante resaltar que la Comisión Interamericana de Derechos Humanos, a través de sus informes, ha interpretado los requisitos de admisibilidad de una petición, los cuales han sido de recibo doctrinal por: Barbosa (2002), Faúndez (2004), Medina \& Nash (2011), Rey (2005), Martín \& Rodríguez (2006), entre otros estudiosos de la materia. No obstante, los trabajos de dichos autores se han centrado en acercar y explicar el funcionamiento del Sistema Interamericano, mas no en la estrategia metodológica de crear espacios convencionales, nichos citacionales y líneas jurisprudenciales.

Esta estrategia metodológica permitirá abordar el objetivo general y los objetivos específicos, es decir, se podrá identificar y analizar de forma precisa el requisito de admisibilidad "no duplicidad de procedimientos internacionales" en sus dos vertientes: no cosa juzgada internacional y no pleito pendiente internacional, requisito último que será objeto de análisis en el presente texto Para que la Comisión pueda declarar inadmisible una petición por la existencia de pleito pendiente internacional, es necesario que se logre demostrar entre las dos peticiones (una tramitada ante la Comisión y la otra tramitada ante otro órgano internacional) la identidad entre los sujetos, el objeto, la base legal y los órganos internacionales (González, 2015).

En relación con los sujetos, lo que se verifica principalmente es la identidad entre los sujetos pasivos y activos de la violación (Corte IDH, 1999, párr. 54; CIDH, 2007a, párr. 70). Sin embargo, es necesario por exigencia reglamentaria de la Comisión verificar también el peticionario y su mandato ante el otro órgano internacional, es decir, el hecho de que sea el mismo peticionario en las dos instancias no lleva o arroja automáticamente la existencia de pleito pendiente internacional (CIDH, 1996, párr. V.A.1).

La Comisión Interamericana al analizar el tema del objeto, ha sido clara en establecer que se está en presencia del marco fáctico de la petición: los hechos (CIDH, 1998, párrs.43, 45 y 46).

En lo referente a la base legal, esta corresponde al marco jurídico de la petición que se encuentra constituido por los derechos, la pretensión y la naturaleza de la denuncia (CIDH, 1998, párrs.43, 45 y 46).

Por último, es importante establecer que la identidad entre los órganos internacionales será objeto de descripción en las siguientes líneas, a través de la identificación, creación y análisis de cinco nichos citacionales.

\section{A. Resultados de la Investigación}

La Convención Americana sobre Derechos Humanos (CADH) consagra en sus artículos 46 y 47 las cláusulas "no pleito pendiente internacional" y "no cosa juzgada internacional", como criterios para la admisibilidad de una petición ante la Comisión Interamericana de Derechos Humanos. Es así como, en su artículo 46 la $\mathrm{CADH}$ establece que "para que una petición o comunicación presentada conforme a los artículos 44 ó 45 sea admitida por la Comisión, se requerirá:[...] que la materia de la petición o comunicación no esté pendiente de otro procedimiento de arreglo internacional[...]" (CADH, 1969),. Seguidamente, el artículo 47 reza de la siguiente manera "la Comisión declarará inadmisible toda petición o comunicación presentada de acuerdo con los artículos 44 ó 45 cuando:[... ] sea sustancialmente la reproducción de petición o comunicación anterior ya examinada por la Comisión u otro organismo internacional[...]" (CADH, 1969).

De forma complementaria, el Reglamento de la Comisión Interamericana de Derechos Humanos 
establece en su artículo 33 la no duplicación de procedimientos en los siguientes términos:

1. La Comisión no considerará una petición si la materia contenida en ella:

a. se encuentra pendiente de otro procedimiento de arreglo ante un organismo internacional gubernamental de que sea parte el Estado en cuestión;

b. reproduce sustancialmente otra petición pendiente o ya examinada y resuelta por la Comisión u otro organismo internacional gubernamental del que sea parte el Estado en cuestión.

2. Sin embargo, la Comisión no se inhibirá de considerar las peticiones a las que se refiere el párrafo 1 cuando:

a. el procedimiento seguido ante el otro organismo se limite a un examen general sobre derechos humanos en el Estado en cuestión y no haya decisión sobre los hechos específicos que son objeto de la petición ante la Comisión o no conduzca a su arreglo efectivo;

b. el peticionario ante la Comisión sea la víctima de la presunta violación o su familiar y el peticionario ante el otro organismo sea una tercera persona o una entidad no gubernamental, sin mandato de los primeros (CIDH, 2009).

De este modo, es un mandato convencional y reglamentario que la Comisión Interamericana no podrá declarar admisible una petición que esté siendo o que ya haya sido considerada por otro órgano internacional. Sin embargo, es pertinente establecer que de lo acordado por los Estados en la Convención Americana y por los comisionados en su reglamento no se pueden extraer los elementos que generan la duplicidad de procedimientos internacionales, ya sea por pleito pendiente o por cosa juzgada; en tanto, estos criterios han sido establecidos tanto por la Corte Interamericana de Derechos Humanos en sus sentencias, como por la Comisión en sus resoluciones e informes.

En otras palabras, los dos órganos de protección de derechos humanos en el contexto del sistema interamericano han indicado que habrá duplicidad de procedimientos internacionales cuando exista la reproducción sustancial de la petición y la misma esté siendo considerada o ya haya sido considerada por la Comisión. Asimismo, han señalado que se está en presencia de la reproducción sustancial cuando exista entre las dos peticiones identidad entre los sujetos, el objeto y la base legal (González, 2015; Corte IDH, 2007, párr. 48; CIDH, 2007a, párr.69).

Ahora bien, además de los tres requisitos anteriores, ambos órganos han interpretado lo que la Convención y el reglamento de la Comisión establecen sobre el mandato relativo a que "la petición no éste siendo o no haya sido conocida por otro órgano internacional". Más claramente, se tiene que tanto la Comisión como la Corte han indicado cuáles son los órganos internacionales que tienen la virtud de producir la duplicidad internacional o, lo que es lo mismo, la inadmisibilidad por la existencia de pleito pendiente o de cosa juzgada.

En la siguiente gráfica, se puede observar el nicho citacional de los pronunciamientos (resoluciones - informes) de la Comisión Interamericana que hacen referencia al requisito "otro organismo internacional gubernamental" como elemento de admisibilidad o inadmisibilidad por la existencia o no de pleito pendiente internacional.

Gráfica 1. Nicho citacional de los pronunciamientos de la CIDH alusivos al requisito "otro organismo internacional gubernamental"

\begin{tabular}{|c|}
\hline Jesús Ángel Gutiérrez Olvera vs. México (CIDH,2010, párrs. 50 y 51) \\
\hline$\downarrow$ \\
\hline Benito Antonio Barrios y Otros vs. Venezuela (CIDH,2009, párrs. 66 y 67) \\
$\downarrow$ \\
\hline Luis Gonzalo "Richard" Vélez Restrepo y familia vs Colombia (CIDH,2008, párrs. 64 y 65) \\
\hline
\end{tabular}




\begin{tabular}{|c|}
\hline \multicolumn{1}{|c|}{$\downarrow$} \\
\hline Wilmer Antonio González Rojas vs. Nicaragua (CIDH, 2007b, párrs. 66 a 68) \\
\hline$\downarrow$ \\
\hline Cecilia Rosana Nuñez Chipana vs. Venezuela (CIDH, 2005a, párrs. 35 a 40) \\
\hline$\downarrow$ \\
\hline Johan Alexis Ortiz Hernández vs. Venezuela (CIDH, 2005b, párrs. 39 a 44) \\
$\downarrow \downarrow$ \\
\hline César Nivia y Vladimir Hincapié Galeano vs. Colombia (CIDH,1995, párrs.25 y 26) \\
\hline$\downarrow$ \\
\hline José Bernardo Díaz y otros vs Colombia (CIDH,1997, párrs. 69, 72, 73 y 76) \\
\hline$\downarrow$ \\
\hline Manuel Stalin Bolaños Quiñonez vs. Ecuador (CIDH, 1995, párr. admisibilidad 2) \\
\hline \\
\hline Orlando García Villamizar y otros vs. Colombia (CIDH, 1992, párr. considerando 1d) \\
\hline$\downarrow$ \\
\hline Juan Gelderes Orozco y Benigno Contreras (CIDH, 1988a, párr. considerando d- h) \\
$\downarrow$ \\
\hline Luis Máximo Vera Aragón (CIDH, 1998b, párr. considerando d- h) \\
\hline Eustaquio Yauli Huaman (CIDH,1988c, párr. considerando d-h) \\
\hline
\end{tabular}

Fuente: elaboración propia tomando en cuenta los pronunciamientos de la Comisión Interamericana de Derechos Humanos entre los años 1988 a 2010

Para la CIDH es de suma importancia que el otro órgano gubernamental internacional que tramite la petición-queja, comunicación, denuncia-sea de aquellos que tenga competencia para adoptar decisiones sobre los hechos específicos contenidos en la petición. La Comisión deja establecido este estándar tanto en sus pronunciamientos -resoluciones e informes- como en su reglamento.

Los casos Eustaquio Yauli Huaman vs. Perú (CIDH, 1988a) y Jesús Ángel Gutiérrez Olvera vs. México (CIDH, 2010), constituyen un ejemplo de lo anterior. En esas oportunidades, los Estados argumentaron y sostuvieron ante la Comisión que la petición debería ser declarada inadmisible por ser tramitada de forma conjunta ante el Grupo de Trabajo sobre Desapariciones Forzadas o Involuntarias de Naciones Unidas. Sin embargo, la Comisión Interamericana siempre ha rechazado este argumento, toda vez que aclara que el órgano citado no tiene competencia para adoptar decisiones sobre los hechos específicos de una petición y no tiene la facultad para adjudicar casos concretos: (CIDH, 1988a, párr. considerando f; CIDH, 2010, párr. 50).
Que la Comisión Interamericana de Derechos Humanos no dispone de ningún antecedente que le permita establecer que la situación de Eustaquio Yauli Huaman ha sido aclarada por el Grupo de Trabajo sobre Desapariciones Forzadas o Involuntarias de la Comisión de Derechos Humanos de Naciones Unidas;

Que en concepto de la Comisión Interamericana de Derechos Humanos, esta sólo debería inhibirse de la consideración del presente caso cuando el asunto se encuentre pendiente de otro procedimiento de arreglo ante una organización internacional gubernamental del que sea parte el Perú y dicho procedimiento sea sustancialmente la reproducción de una petición pendiente o ya examinada y resuelta por la Comisión u otro organismo internacional gubernamental del que sea parte el Perú;

Que, en cambio, la Comisión no debe inhibirse de conocer de la presente situación cuando el procedimiento seguido ante la otra organización se limita al examen de la 
situación general sobre derechos humanos en un Estado, y no exista una decisión sobre los hechos específicos que son objeto de la petición sometida a la Comisión o que no conduzca a un arreglo efectivo de la violación denunciada;

Que, de conformidad con las pertinentes resoluciones de la Comisión de Derechos Humanos de Naciones Unidas, en particular, la Resolución 20 (XXVI) de 29 de febrero de 1980 , no se encuentra dentro del mandato del Grupo de Trabajo sobre Desapariciones Forzadas o Involuntarias decidir respecto a los hechos específicos que se han alegado en el presente caso;
Que, en consecuencia, la Comisión Interamericana de Derechos Humanos no se encuentra inhibida para conocer el presente caso de acuerdo con la Convención Americana sobre Derechos Humanos y su Reglamento [...] (CIDH, 1988a, párr. considerando d al h).

En la gráfica a continuación, se puede observar el nicho citacional que podría generarse desde el espacio convencional denominado "para que haya pleito pendiente internacional se requiere que la petición esté siendo considerada por un organismos internacional que tenga competencia para adoptar decisiones sobre los hechos específicos contenidos en la petición".

Gráfica 2. Nicho citacional desde el espacio convencional denominado "para que haya pleito pendiente internacional se requiere que la petición esté siendo considerada por un organismos internacional que tenga competencia para adoptar decisiones sobre los hechos específicos contenidos en la petición”.

\begin{tabular}{|c|}
\hline Jesús Ángel Gutiérrez Olvera vs. México (CIDH,2010, párr. 50) \\
\hline$\downarrow$ \\
\hline Benito Antonio Barrios y Otros vs. Venezuela (CIDH, 2009, párr. 66) \\
\hline$\downarrow$ \\
\hline Luis Gonzalo "Richard" Vélez Restrepo y familia vs Colombia (CIDH, 2008, párr. 64) \\
\hline$\downarrow$ \\
\hline Cecilia Rosana NuñezChipanavs. Venezuela (CIDH, 2005a, párr. 37) \\
\hline \\
\hline Johan Alexis Ortiz Hernandezvs. Venezuela (CIDH, 2005b, párr. 39) \\
\hline$\downarrow$ \\
\hline César Nivia y Vladimir Hincapíe Galeano vs. Colombia (CIDH, 1995, párr. 25) \\
\hline$\downarrow$ \\
\hline Eustaquio Yauli Huaman (CIDH, 1988c, párr. considerando f) \\
\hline$\downarrow$ \\
\hline Luis Máximo Vera Aragón (CIDH, 1998b, párr. considerando f) \\
\hline
\end{tabular}

Fuente: elaboración propia tomando en cuenta los pronunciamientos de la Comisión Interamericana de Derechos Humanos entre los años 1988 a 2010. 
De igual manera, la Comisión Interamericana determinó que para que una petición sea declarada inadmisible por pleito pendiente internacional es necesario que el órgano donde se tramita de forma paralela la petición tenga la competencia para adoptar medidas tendientes a su efectiva resolución, de lo contrario la petición será admisible.

En el caso César Chaparro Nivia y Vladimir Hincapié Galeano, Colombia solicitó a la CIDH que declarará la petición inadmisible, toda vez que la situación de tortura de las víctimas estaba siendo estudiada tanto por el Relator sobre la Cuestión de la Tortura como por el Relator Especial sobre Ejecuciones Extrajudiciales, Sumarias o Arbitrarias, ambos pertenecientes al Sistema de las Naciones Unidas, lo cual generaba duplicidad internacional. No obstante,, la Comisión no aceptó el argumento estatal, y reiteró su posición al respecto así:

En efecto, según señala el artículo 39(2) (a) del Reglamento de la Comisión, ésta no se debe inhibir de conocer y examinar una petición relativa a un caso ante otra organización internacional gubernamental de la que sea parte el Estado aludido toda vez que: El procedimiento seguido ante la otra organización u organismo se limite al examen de la situación general de derechos humanos en el Estado aludido y no exista una decisión sobre los hechos específicos que son objeto de la petición sometida a la Comisión que no conduzca a un arreglo efectivo de la violación denunciada.
Esto significa que no existe duplicación toda vez que el procedimiento seguido ante el otro ente no conduzca a una decisión sobre la violación de que se trate. La jurisprudencia de Comisión confirma esta interpretación.

La Comisión considera, en virtud de esta norma, que el Relator y el Grupo de Trabajo de las Naciones Unidas antes mencionados no pertenecen a la categoría de órganos internacionales cuyo mandato pueda generar la duplicación a la que se refieren los artículos 46(1)(c) y 47(1)(d) de la Convención Americana. En efecto, se trata de mecanismos que no pueden conducir al arreglo efectivo de la violación denunciada. Además, el Estado no ha presentado antecedentes que permitan establecer que la situación de las víctimas en este caso haya sido aclarada por estos entes. Por lo anteriormente expuesto, la Comisión entiende que los requisitos establecidos en los artículos 46(1)(c) y 47(1)(d) se encuentran satisfechos (CIDH, 1999, párrs. 25 y 26).

La gráfica que sigue permite observar el nicho citacional que podría generarse desde el espacio convencional denominado "para que haya pleito pendiente internacional se requiere que la petición esté siendo considerada por un organismo internacional que tenga competencia para adoptar medidas tendientes a la efectiva resolución de la disputa de que se trate".

Gráfica 3. Nicho citacional desde el espacio convencional denominado "para que haya pleito pendiente internacional se requiere que la petición esté siendo considerada por un organismos internacional que tenga competencia para adoptar medidas tendientes a la efectiva resolución de la disputa de que se trate".

\begin{tabular}{|c|}
\hline Jesús Ángel Gutiérrez Olvera vs. México (CIDH, 2010, párr. 50) \\
\hline$\downarrow$ \\
\hline Benito Antonio Barrios y Otros vs. Venezuela (CIDH, 2009, párr. 66) \\
\hline$\downarrow$ \\
\hline Luis Gonzalo "Richard" Vélez Restrepo y familia vs Colombia (CIDH, 2008, párr. 64) \\
\hline$\downarrow$ \\
\hline
\end{tabular}




\begin{tabular}{|c|}
\hline Cecilia Rosana Nuñez Chipana vs. Venezuela (CIDH, 2005a, párr. 37) \\
\hline$\downarrow$ \\
\hline César Nivia y Vladimir Hincapíe Galeano vs. Colombia (CIDH, 1995, párr. 26) \\
\hline$\downarrow$ \\
\hline Juan Gelderes Orozco y Benigno Contreras (CIDH, 1988a, considerando f) \\
\hline$\downarrow$ \\
\hline Luis Máximo Vera Aragón (CIDH, 1998b, considerando f) \\
\hline$\downarrow$ \\
\hline Eustaquio Yauli Huaman (CIDH, 1988c, considerando f) \\
\hline
\end{tabular}

Fuente: elaboración propia tomando en cuenta los pronunciamientos de la Comisión Interamericana de Derechos Humanos entre los años 1988 a 2010.

La CIDH en sus pronunciamientos ha establecido que habrá pleito pendiente internacional cuando se logre demostrar que el mandato del otro órgano internacional es de naturaleza convencional; criterio que fue reiterado en el caso Johan Alexis Ortiz Hernández, en el cual Venezuela solicita que se declare el pleito como no admisible, debido a que el mismo está siendo tramitado ante el Relator Especial sobre Ejecuciones Extrajudiciales, Sumarias o Arbitrarias de las Naciones Unidas. Sin embargo, la Comisión desestimó la solicitud manifestando que tanto la naturaleza del mandato de los dos órganos, como la naturaleza de su procedimiento eran disimiles, debido a que el mandato del Relator deviene de una Resolución del Consejo Económico y Social de Naciones Unidas, mas no de una norma convencional, como es el caso de la Comisión.

En el presente caso, tanto el origen y contenido del mandato de la Comisión Interamericana de Derechos Humanos así como la naturaleza del procedimiento ante la misma son diferentes a los del Relator Especial sobre ejecuciones extrajudiciales, sumarias $\mathrm{O}$ arbitrarias de las Naciones Unidas.

El mandato del Relator Especial sobre ejecuciones extrajudiciales, sumarias o arbitrarias de Naciones Unidas fue establecido en virtud de la resolución 1982/35 del Consejo Económico y Social de Naciones Unidas. El mandato de la Comisión Interamericana de
Derechos Humanos en este caso deriva de una fuente convencional, la Convención Americana sobre Derechos Humanos. La Comisión, a diferencia del Relator Especial sobre ejecuciones extrajudiciales, sumarias o arbitrarias, pertenece a la categoría de órganos de supervisión de tratados.

La Comisión concluye por tanto que el Relator Especial sobre ejecuciones extrajudiciales, sumarias o arbitrarias de las Naciones Unidas no pertenece a la categoría de órganos internacionales cuyo mandato pueda generar la duplicación a la que se refieren los artículos 46(1)(c) y 47(d) de la Convención Americana (CIDH, 2005b, párrs. 40, 41 y 44).

De igual forma, la CIDH ha establecido que una petición será inadmisible cuando el procedimiento seguido ante el otro órgano internacional se caracterice -igual que el de la Comisión por ser contradictorio o contencioso. Además, es necesario que el órgano tenga un rol adjudicativo dentro del procedimiento, criterio que fue confirmado, en los casos Jesús Ángel Gutiérrez Olvera us. México y Wilmer Antonio González Rojas vs. Nicaragua, entre otros.

En relación con el caso Wilmer Antonio González Rojas, Nicaragua trató de demostrar ante la Comisión Interamericana que al declarar su admisibilidad se estaría violando el principio non bis in ídem, debido a que la petición fue previamente 
objeto de conocimiento por parte del Grupo de Trabajo sobre Situaciones de la Comisión de Derechos Humanos de las Naciones Unidas en el año 2001, la cual decidió dar por finalizado el asunto (CIDH, 2007, párr. 65). A pesar de esta argumentación la Comisión consideró que:

La Comisión considera que la naturaleza de las dos instancias internacionales es diferente, y por tanto, no hay duplicación. El procedimiento 1503 de Naciones Unidas no es un mecanismo convencional, no tiene la facultad de adjudicar casos concretos y el procedimiento de comunicaciones individuales ante el mismo no está orientado a una solución de naturaleza contenciosa. Este procedimiento, sin carácter contradictorio, $y$ confidencial se limita a solicitar al gobierno interesado aclaraciones sobre las denuncias. El procedimiento ante el Sistema Interamericano de Protección de Derechos Humanos por su parte es de naturaleza convencional y carácter contencioso, y la Comisión Interamericana sí tiene un rol adjudicativo dentro de ese procedimiento. El procedimiento ante la Comisión Interamericana puede concluir con una serie de recomendaciones que los Estados deben cumplir de buena fe.

La Comisión concluye por tanto que el procedimiento 1503 de Naciones Unidas no pertenece a la categoría de órganos internacionales cuyo mandato pueda generar la duplicación a la que se refieren los artículos 46.1.c y 47.d de la Convención Americana (CIDH, 2007, párrs. 67 y 68).

Este argumento fue también reseñado en el caso Jesús Ángel Gutiérrez Olvera, en el cual la Comisión Interamericana desestimó la solicitud de México de inadmisibilidad, indicando queel Grupo de Trabajo sobre Desapariciones Forzadas e Involuntarias de Naciones Unidas no tiene la facultad de adjudicar casos concretos. Además, aunque se trata de un mecanismo en el que se pueden plantear situaciones concretas de desapariciones no tiene un sistema de casos que tenga como objetivo emitir decisiones que atribuyan responsabilidades específicas. En consecuencia, el procedimiento del Grupo de Trabajo es principalmente de naturaleza humanitaria y carece de carácter contradictorio (CIDH, 2010, párr. 50).

En la siguiente gráfica, se puede observar el nicho citacional que podría generarse desde el espacio convencional denominado "para que haya pleito pendiente internacional se requiere que la petición esté siendo considerada por un organismo internacional cuyo procedimiento sea de naturaleza convencional y de carácter contencioso, contradictorio y rol adjudicativo".

Gráfica 4. Nicho citacional desde el espacio convencional denominado "para que haya pleito pendiente internacional se requiere que la petición esté siendo considerada por un organismo internacional cuyo procedimiento sea de naturaleza convencional y de carácter contencioso, contradictorio y rol adjudicativo".

\begin{tabular}{|c|}
\hline Jesús Ángel Gutiérrez Olvera vs. México (2010, párr. 50) \\
\hline$\downarrow$ \\
\hline Wilmer Antonio González Rojas vs. Nicaragua (2007, párr. 67) \\
\hline$\downarrow$ \\
\hline Johan Alexis Ortiz Hernández vs. Venezuela (2005b, párr. 40 a 43) \\
\hline
\end{tabular}

Fuente: elaboración propia tomando en cuenta los pronunciamientos de la Comisión Interamericana de Derechos Humanos entre los años 1988 a 2010.

Por último, es necesario también tener presente que la $\mathrm{CIDH}$ ha establecido que no habrá inadmisibilidad cuando el caso se tramite de forma simultánea en un procedimiento ante otra instancia internacional la cual sólo se limite a hacer un examen general sobre la situación de los derechos humanos del país denunciado. De manera que la Corte confirma el criterio ya indicado según el cual mientras el examen del caso no sea sobre los hechos específicos y la 
decisión final del órgano no sea adjudicativa no es procedente declarar su inadmisibilidad.

Este pronunciamiento ha sido reiterado en diversos casos, en los cuales se tramitaba la petición de forma conjunta o simultánea ante órganos de Naciones Unidas como el Grupo de Trabajo sobre Desapariciones Forzadas o Involuntarias, la Comisión de Derechos Humanos, el Relator sobre la Cuestión de la Tortura y el Relator Especial sobre Ejecuciones Extrajudiciales, Sumarias o Arbitrarias. En síntesis, en todos ellos la Comisión Interamericana declaró la admisibilidad señalando que son órganos que no deciden sobre los hechos específicos, sino que su facultad se limita a hacer un análisis general de la situación de derechos humanos en el Estado.

En efecto, según señala el artículo 39(2) (a) del Reglamento de la Comisión, ésta no se debe inhibir de conocer y examinar una petición relativa a un caso ante otra organización internacional gubernamental de la que sea parte el Estado aludido toda vez que: El procedimiento seguido ante la otra organización u organismo se limite al examen de la situación general de derechos humanos en el Estado aludido y no exista una decisión sobre los hechos específicos que son objeto de la petición sometida a la Comisión que no conduzca a un arreglo efectivo de la violación denunciada.

Esto significa que no existe duplicación toda vez que el procedimiento seguido ante el otro ente no conduzca a una decisión sobre la violación de que se trate. La jurisprudencia de Comisión confirma esta interpretación.

La Comisión considera, en virtud de esta norma, que el Relator y el Grupo de Trabajo de las Naciones Unidas antes mencionados no pertenecen a la categoría de órganos internacionales cuyo mandato pueda generar la duplicación a la que se refieren los artículos 46(1)(c) y 47(1)(d) de la Convención Americana. En efecto, se trata de mecanismos que no pueden conducir al arreglo efectivo de la violación denunciada. Además, el Estado no ha presentado antecedentes que permitan establecer que la situación de las víctimas en este caso haya sido aclarada por estos entes. Por lo anteriormente expuesto, la Comisión entiende que los requisitos establecidos en los artículos 46(1)(c) y 47(1)(d) se encuentran satisfechos (CIDH, 1999, párrs. 25 y 26).

En el caso José Bernardo Díaz y otros, Colombia le solicita a la $\mathrm{CIDH}$ que lo declare inadmisible, argumentando que ese mismo órgano en su Segundo Informe sobre la Situación de los Derechos Humanos en Colombia, ya había abordado alegaciones en relación con la persecución política de miembros de la Unión Patriótica (CIDH, 1997, párr. 68). Sin embargo, la Comisión desestimó el argumento estatal e indicó:

Ni la Convención ni el Reglamento de la Comisión requieren que la Comisión declare la inadmisibilidad de un caso porque el objeto del caso se haya planteado anteriormente en un informe general. De hecho, el Reglamento de la Comisión, artículo 19(2)(b) establece, en lo pertinente, que miembros de la Comisión no podrán discutir o decidir un asunto sometido a la consideración de la Comisión sólo "si previamente hubiesen participado, a cualquier título, en alguna decisión sobre los mismos hechos" (énfasis agregado). La discusión de hechos específicos en un informe general sobre un país no constituye una "decisión" sobre dichos hechos, como sí lo constituiría un informe final sobre una petición individual, en la cual se han denunciado los mismos o similares hechos.

[...]La tramitación de un caso con arreglo al procedimiento de petición individual es más estructurada que la preparación de un informe general, que cumple un papel informativo en vez de adjudicatorio. La tramitación de una petición individual exige que la Comisión siga los procedimientos enunciados en los artículos 44 al 51 de la Convención. La Comisión debe realizar un 
análisis exhaustivo del caso a los efectos de llegar a conclusiones de hecho y de derecho, conforme a las disposiciones de los artículos 50 y 51 de la Convención.

[...] Por otra parte, la Comisión es de la opinión de que debe estar en condiciones de incluir información sobre situaciones concretas de derechos humanos en los informes generales relacionados con los Estados miembros de la Organización de los Estados Americanos. La Comisión debe poder incluir información aunque esté relacionada con un caso ya abierto o con uno que podría presentarse en el marco del sistema de peticiones individuales. De otro modo, se vería obligada e excluir de sus informes generales sobre los países la consideración de segmentos enteros del panorama de los derechos humanos en esos países.

[...] Por último, la Comisión desea señalar que ha admitido y resuelto casos presentados por la vía del sistema de peticiones individuales y que a la vez ha decidido incluir información sobre los temas de los casos en sus informes generales. La falta de objeción ha convertido a estas decisiones repetidas de la Comisión en una práctica aceptada por los Estados miembros de la Organización de los Estados Americanos. La Corte también ha indicado que la Comisión puede publicar en sus informes generales información sobre la situación de los derechos humanos y a la vez decidir, o elevar a la Corte, un caso individual que esté relacionado con la misma situación. La Comisión no acepta la excepción del Estado sobre inadmisibilidad fundada en el hecho de que la Comisión ha hecho un análisis previo del caso (CIDH, 1997, párrs. 69, 72, 74 y 76).

Del anterior caso se puede concluir que lo más relevante para la Comisión es la naturaleza del procedimiento, más no que el mandato y el órgano sean convencionales. De modo que, de acuerdo con la $\mathrm{CIDH}$, es fundamental que el procedimiento sea convencional, contradictorio, contencioso, así como, que la decisión final de ese procedimiento sea adjudicativa y no se limite a un examen general de la situación de derechos humanos en el Estado.

Para efectos prácticos, se puede observar en la gráfica enseguida el nicho citacional que podría generarse desde el espacio convencional denominado "para que haya pleito pendiente internacional se requiere que la petición esté siendo considerada por un organismo internacional, cuyo procedimiento no se limite a un examen general sobre derechos humanos en el Estado denunciado".

Gráfica 5. Nicho citacional desde el espacio convencional denominado "para que haya pleito pendiente internacional se requiere que la petición esté siendo considerada por un organismo internacional, cuyo procedimiento no se limite a un examen general sobre derechos humanos en el Estado denunciado".

\begin{tabular}{|c|}
\hline Johan Alexis Ortiz Hernández vs. Venezuela (CIDH, 2005b, párr. 39) \\
\hline$\downarrow$ \\
\hline César Nivia y Vladimir Hincapíe Galeano vs. Colombia (CIDH,1995, párr. 25) \\
$\downarrow$ \\
\hline José Bernardo Díaz y otros vs Colombia (CIDH,1997, párr. 69) \\
\hline$\downarrow$ \\
\hline Manuel Stalin Bolaños Quiñonez vs. Ecuador (CIDH, 1995, párr. admisibilidad 2) \\
\hline$\downarrow$ \\
\hline
\end{tabular}




\begin{tabular}{|c|}
\hline Juan Gelderes Orozco y Benigno Contreras (CIDH, 1988a, párr. considerando f) \\
\hline$\downarrow$ \\
\hline Luis Máximo Vera Aragón (CIDH, 1998b, párr. considerando f) \\
\hline$\downarrow$ \\
\hline Eustaquio Yauli Huaman (CIDH, 1988c, párr. considerando f) \\
\hline
\end{tabular}

Fuente: elaboración propia tomando en cuenta los pronunciamientos de la Comisión Interamericana de Derechos Humanos entre los años 1988 a 2010.

\section{CONCLUSIONES}

Queda claro con la lectura de la Convención Americana sobre Derechos Humanos y del Reglamento de la Comisión Interamericana de Derechos Humanos que la Comisión podrá declarar admisible una petición cuando su materia no esté pendiente de otro procedimiento de arreglo internacional. Asimismo, la duplicidad de procedimientos internacionales se puede generar por la existencia de "pleito pendiente internacional" o "de cosa juzgada internacional"; siendo necesario en los dos eventos entrar a analizar si existe identidad entre los sujetos, objeto, base legal y órganos; requisitos que son identificados de la recolección, lectura y análisis de los pronunciamientos de la CIDH desde 1988 a 2010.

En relación con los sujetos, la Comisión Interamericana de Derechos Humanos verifica si existe identidad entre las víctimas, peticionarios y Estado. En cuanto al objeto, estudia el marco fáctico de la petición -hechos-. Por lo que se refiere a la base legal, analiza el marco jurídico de la petición derechos, pretensión y naturaleza de la denuncia.

Por último, con respecto al órgano, la CIDH valora la correspondencia entre su propio mandato y el mandato del otro órgano internacional que tramita la petición-comunicación, queja o denuncia; así como el procedimiento seguido ante ella y el procedimiento seguido ante el otro órgano de internacional. Por consiguiente,para que se genere pleito pendiente internacional, además de existir identidad entre los sujetos, objeto y base legal, debe existir identidad (correspondencia) entre los dos órganos internacionales en los siguientes aspectos: (1) los dos órganos deben tener competencia para adoptar decisiones sobre los hechos específicos contenidos en la petición, (2) los dos órganos deben tener competencia para adoptar medidas tendientes a la efectiva resolución de la disputa de que se trate, (3) los dos órganos deben tener un mandato establecido convencionalmente, (4) el procedimiento ante los dos órganos debe caracterizarse por ser contencioso, (5) el procedimiento ante los dos órganos debe caracterizarse por ser contradictorio y (6) los dos órganos deben tener un rol adjudicativo.

No obstante, ante la plena identidad entre los sujetos, el objeto, la base legal y los órganos de las dos peticiones que se tramitan de forma conjunta, es importante señalar que el sólo tramite no lleva de forma automática a la inadmisibilidad de la petición.

Por lo tanto, si una petición ya ha sido fallada de fondo por el otro órgano, la Comisión Interamericana estará facultada para declarar la duplicidad internacional por cosa juzgada internacional. Por otra parte, si la petición ha sido fallada de forma - cuestiones de admisibilidad-por el otro órgano internacional, la Comisión estará facultada para declarar la duplicidad internacional por pleito pendiente internacional.

Sin embargo, es importante resaltar que ante la existencia de un trámite conjunto de denuncias y ante la existencia de identidad de sujetos, objeto, base legal y órgano, si éste último -el otro órgano internacional-no se ha pronunciado en cuanto a la admisibilidad de la petición, la Comisión no estará facultada para declarar la inadmisibilidad por pleito pendiente internacional. 
Ahora bien, lo que queda por relacionar en otro producto de la investigación, a través de la deducción y la descripción que se ha hecho es:ciprospera la duplicidad de procedimientos, sea por pleito pendiente o por cosa juzgada, si se logra demostrar que un Estado tiene como práctica generalizada, propia y única el aceptar de buena fe las recomendaciones que le hacen los órganos de derechos humanos de naturaleza no convencional?

\section{REFERENCIAS}

Barbosa, F. (2002). Litigio Interamericano: perspectiva jurídica del sistema de protección de derechos humanos. Bogotá D.C.: Universidad Jorge Tadeo Lozano.

Comisión Interamericana de DDHH Resolución No 7/88. Caso 9504. Inadmisibilidad. Eustaquio Yauli Huaman vs. Perú. (1988c).

Comisión Interamericana de DDHH ResoluciónN ${ }^{\circ}$ 30/88. Caso9748. Inadmisibilidad. Luis Máximo Vera Aragón Vs. Perú. (1988b).

Comisión Interamericana de DDHH ResoluciónN ${ }^{\circ}$ 33/88. Caso9786. Inadmisibilidad. Juan Geldres Orozco y Benigno Contreras Vs. Perú. (1988 a).

Comisión Interamericana de DDHH Informe núm. 1/92. Caso 10.235. Fondo. Orlando García Villamizar, Pedro Pablo Silva Bejarano, Rodolfo Espitia Rodríguez, Edgar Helmut García Villamizar, Gustavo Campos Guevara, Hernando Ospina Rincón, Rafael Guillermo Prado J., Edilbrando Joya Gómez, Francisco Antonio Medina, Bernardo Heli Acosta Rojas y Manuel Darío Acosta Rojas Vs. Colombia. (1992).

Comisión Interamericana de DDHH Informe núm. 10/95. Caso 10.580. Fondo. Manuel Stalin Bolaños Quiñonez Vs. Ecuador. (1995).

Comisión Interamericana de DDHH Informe núm. 5/96. Caso 10.970. Fondo. Raquel Martín De Mejía Vs. Perú. (1996).
Comisión Interamericana de DDHH Informe núm. 5/97. Caso 11.227. Admisibilidad. José Bernardo Díaz y otrosVs. Colombia. (1997).

Comisión Interamericana de DDHH Informe núm. 96/98Caso 11.827. Inadmisibilidad. Peter Blaine Vs. Jamaica. (1998).

Comisión Interamericana de DDHH Informe núm. 30/99. Caso 11.026. Admisibilidad. César Chaparro Nivia y Vladimir Hincapié Galeano Vs. Colombia. (1999).

Comisión Interamericana de DDHH Informe núm. 89/05. (2005). Petición 12.103. Inadmisibilidad. Cecilia Rosana Nuñez Chipana Vs. Venezuela. $\left(2005^{\mathrm{a}}\right)$.

Comisión Interamericana de DDHH Informe núm. 22/05. Petición 12.270. Admisibilidad. Johan Alexis Ortiz HernándezVs. Venezuela. (2005b).

Comisión Interamericana de DDHH Informe núm. 41/07. Petición 998-05. Admisibilidad. Lazinho Brambilla Da Silva Vs. Brasil.(2007).

Comisión Interamericana de DDHH Informe núm. 54/07. (2007). Petición 4614-02. Admisibilidad. Wilmer Antonio González Rojas Vs. Nicaragua. (2007).

Comisión Interamericana de DDHH Informe núm. 47/08. Petición 864-05. Admisibilidad. Luis Gonzalo "Richard" Vélez Restrepo y Familia Vs. Colombia. (2008).

Comisión Interamericana de DDHH Informe núm. 01/09. Petición 1491-05. Admisibilidad. Benito Antonio Barrios y Otros Vs. Venezuela. (2009).

Comisión Interamericana de DDHH Informe núm. 147/10. Petición 497-03. Admisibilidad. Jesús Ángel Gutiérrez Olvera Vs. México. (2010).

Corte Interamericana de Derechos Humanos. Caso Baena Ricardo y otros Vs. Panamá. Excepciones Preliminares. Serie C No. 61. Sentencia del 18 de noviembre de 1999. 
Corte Interamericana de Derechos Humanos. Caso del Pueblo Saramaka. Vs. Surinam. Excepciones Preliminares, Fondo, Reparaciones y Costas. Serie C No. 172. Sentencia de 28 de noviembre de 2007.

Convención Americana sobre Derechos Humanos. Suscrita en San José de Costa Rica en la Conferencia Especializada Interamericana sobre Derechos Humanos. Artículos 46-48. Noviembre 22 de 1969.

Faúndez, H. (2004). El Sistema Interamericano de Protección de los Derechos Humanos. Aspectos Institucionales y Procedimentales. San José de Costa Rica: Instituto Interamericano de Derechos Humanos.

González, A (2015). Pleito Pendiente Internacional. Una mirada desde la Comisión Interamericana de Derechos Humanos.Revista Justicia, 27. Trabajo en trámite de publicación.
López, D. (2008). El derecho de los jueces. Bogotá D.C.: Legis.

Martin, C. \& Rodríguez, D. (2006). La prohibición de la tortura y los malos tratos en el Sistema Interamericano Manual para víctimas y sus defensores. Ginebra-Suiza: Organización Mundial contra la Tortura.

Medina, C. \& Nash, C. (2011). Sistema Interamericano de Derechos Humanos: Introducción a sus Mecanismos de Protección. Santiago de Chile: Universidad de Chile.

Reglamento de la Comisión Interamericana De Derechos Humanos. Aprobado por la Comisión en su $137^{\circ}$ período ordinario de sesiones, artículos 26, 28 y 33, celebrado del 28 de octubre al 13 de noviembre de 2009.

Rey, E. \& Rey, M. (2006). Medidas Provisionales y Medidas Cautelares en el Sistema Interamericano de Derechos Humanos. Bogotá D.C.: Temis. 